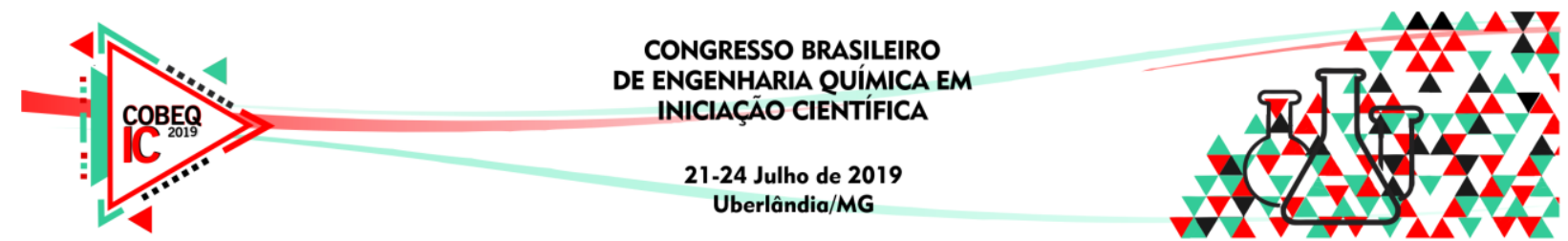

\title{
INVESTIGAÇÃO DO SINAL AMPEROMÉTRICO NA PAREDE EXTERNA DE UM BIOSSENSOR DO TIPO DISCO DE BIOFILME POROSO
}

\author{
E. C. S. SANTOS ${ }^{1}$, L. S. GOMES ${ }^{1}$, M. V. SOUZA JÚNIOR ${ }^{1}$, S. A. CARDOSO ${ }^{1}$ \\ ${ }^{1}$ Universidade Federal do Pará, Faculdade de Biotecnologia \\ E-mail para contato: ecsantos20@gmail.com
}

\begin{abstract}
RESUMO - Foi realizada a investigação computacional do desenvolvimento de um biossensor amperométrico em formato de disco, acoplado a um biofilme à base de amido de mandioca (Manihot esculenta), utilizando enzimas imobilizadas do tipo tirosinase para o auxílio no processo de reação da membrana com o substrato. O software COMSOL MULTIPHISICS foi utilizado na simulação com o intuito de estudar as equações provenientes dos balanços realizados no biossensor. O dispositivo foi estudado contendo duas membranas, uma sem enzima, contendo apenas o biofilme poroso, e na segunda membrana a presença de um biofilme poroso contendo a enzima imobilizada, representando um processo difusivo sem reação e com reação, respectivamente. Os resultados obtidos a partir dos estudos feitos no software se demonstraram satisfatórios quanto à influência da porosidade no processo de difusão do substrato na membrana biopolimérica porosa, contendo a enzima imobilizada, além de contribuir para o estudo futuro de outros parâmetros, que venham contribuir na otimização do sinal de detecção do biossensor.
\end{abstract}

Palavras-chave: biossensor, enzimas, biofilme, porosidade.

\section{INTRODUÇÃO}

Os biossensores são dispositivos nos quais uma enzima ou uma proteína são utilizadas para medir de modo seletivo determinadas substâncias em um meio específico (OLIVEIRA, RIBEIRO, et al, 2015), ou seja, são pequenos dispositivos que utilizam reações biológicas para detecção de analitos alvo, combinando um componente biológico que interage com um substrato-alvo a um transdutor físico, convertendo os processos de reconhecimento em sinais mensuráveis (CALIL, et al, 2015). A utilização desse tipo de sensor traz muitas vantagens, entre elas a alta sensibilidade e seletividade destes, como também a facilidade em termos de desenvolvimento do sensor, sendo assim acessíveis e prontos para o uso. Além disso, no processos de simulação realizada no software COMSOL Multiphisics, foram utilizadas as reações de Langmüir, Lei de Fick, Lei de Faraday, cinética enzimática de Michaeles-Menten, além dos balanços de substrato e produto para a realização desse processo.

Portanto, o referido estudo trata da simulação de um biossensor, que tem como enzima a tirosinase, obtida de diversas fontes como o buriti, que catalisa etapas de oxidação sequenciais na presença de vários substratos fenólicos (CARDOSO, 2014), junto a um biofilme poroso gerado a partir do amido da mandioca (Manihot esculenta), com o intuito de analisar os fenóis presentes em efluentes urbanos utilizando nano partículas de cobre como eletrodo de trabalho.

\section{MODELAGEM MATEMÁTICA DO PROBLEMA}

Um processo típico de transferência de massa em um biossensor é apresentado na Figura 1.1. No esquema da figura abaixo, a enzima escolhida foi a tirosinase, que será utilizada para a detecção de compostos fenólicos em efluentes urbanos. 


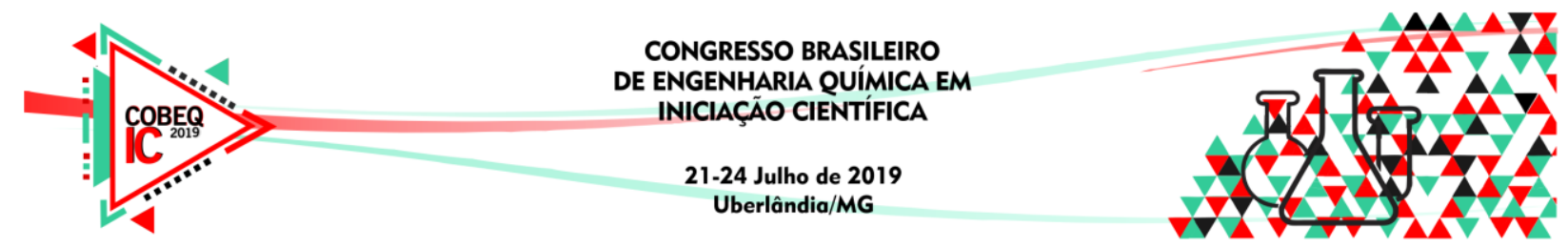

Figura 1.1 - Representação de um biossensor poroso esquematizado.

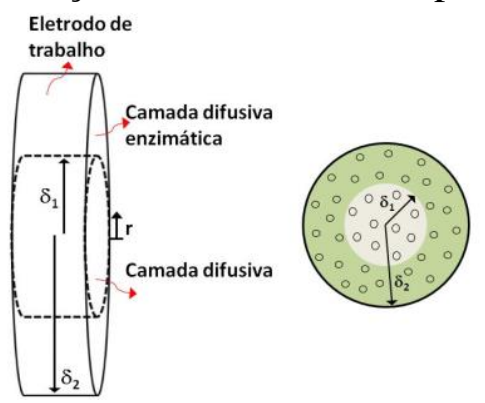

A substância fenólica presente no substrato (S) migra por difusão através da membrana circular porosa 1, até encontrar a superfície da membrana circular porosa 2, a qual apresenta enzimas imobilizadas em sua estrutura (E). A substância fenólica reage com a enzima conforme a reação abaixo:

$S+E \rightarrow P_{1}$

O produto $\left(\mathrm{P}_{1}\right)$ formado na membrana circular porosa 2 , migra por difusão até a superfície do eletrodo de trabalho, formado por nano partículas de cobre, onde reage com as nano partículas através de uma reação de oxirredução para formar o produto $\left(\mathrm{P}_{2}\right)$ e elétrons livres ( $\overline{\mathrm{e}})$, reação abaixo:

$P_{1}+C u^{0} \rightarrow P_{2}+\bar{e}$

Os elétrons produzidos irão migrar devido um gradiente de elétrons através de um circuito eletrônico simples, até a extremidade onde se encontra um medidor de corrente elétrica, amperímetro. Esse sinal elétrico sentido pelo amperímetro será convertido em concentração de fenóis através de relações matemáticas do balanço de espécies do processo de difusão presente no biossensor. Dessa forma, foram balanços de espécies nas membranas circulares porosas 1 e 2 para as espécies substrato e produto.

\subsection{Balanço na Membrana circular 1: Substrato}

$P_{1} \frac{\partial C_{s 1}}{\partial t}-\nabla\left(D_{s 1} C_{s 1}\right)=0 \quad t>0 \quad 0<r<\delta_{1}$

$C_{s 1}(r, t=0)=S_{0} \quad t=0 \quad 0<r<\delta_{1} ; \frac{\partial C_{s 1}}{\partial r}=0 \quad r=0 ; \quad \frac{\partial C_{s 1}}{\partial r}=0 \quad r=\delta_{1} \quad t>0$

$P_{1}=\varepsilon_{1}+\rho k_{p 1}$

onde $\mathrm{C}_{\mathrm{s} 1}(\mathrm{r}, \mathrm{t})$ é o perfil de concentração de efluente ao longo do raio da membrana circular porosa 1 e é medida por mol. $\mathrm{m}^{-3} ; \mathrm{S}_{0}$ é a concentração inicial de efluente e medida por mol.m ${ }^{-}$ ${ }^{3} ; \delta_{1}$ é o raio da membrana circular porosa 1 e medido em $\mathrm{m}$; $\mathrm{D}_{\mathrm{s} 1}$ é o coeficiente de difusão do efluente na membrana circular porosa 1 e é medido $\mathrm{em} \mathrm{m}^{2} . \mathrm{s}^{-1} ; \mathrm{P}_{1}$ representa a porosidade na membrana $1 ; \varepsilon_{1}$ é também a porosidade na membrana 1 , é medido em $1 \mu \mathrm{m}$; e $\rho$ é a massa específica do biofilme, que equivale a $1400 \mathrm{~kg} / \mathrm{m}^{3}$. A equação de Langmuir é ser definida como:

$q_{s 1}(r, t)=\frac{C_{s 1, \max }^{*} K_{L, s 1}}{1+C_{s 1}^{*}(r, t) K_{L, s 1}}$ 


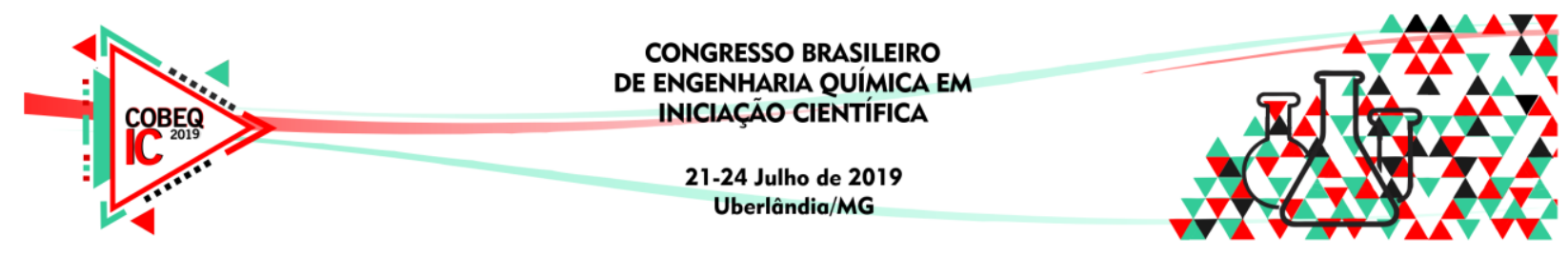

onde, $\mathrm{q}_{\mathrm{s} 1}(\mathrm{r}, \mathrm{t})$ é a concentração de substrato adsorvido por unidade de massa de adsorvente, em $\mathrm{g} / \mathrm{g} ; \mathrm{C}_{\mathrm{s} 1}{ }^{*}(\mathrm{r}, \mathrm{t})$ é a concentração no equilíbrio do substrato em solução, em mol.m ${ }^{-3} ; \mathrm{C}^{*}{ }_{\mathrm{s} 1 \text {,max }}$ é o parâmetro que representa a concentração máxima de adsorção na monocamada, em mol.m ${ }^{3}$; $K_{L, s l}$ é o parâmetro relacionado com a energia de adsorção e dependente da temperatura, medido em $1 . \mathrm{g}^{-1}$.

\subsection{Balanço na Membrana circular 2: Substrato}

$$
\begin{aligned}
& P_{2} \frac{\partial C_{s 2}}{\partial t}-\nabla\left(D_{s 2} C_{s 2}\right)=-R_{s} \quad t>0 \quad \delta_{1}<r<\delta_{2} \\
& C_{s 2}(r, t=0)=0 \quad t=0 \quad \delta_{1}<r<\delta_{2} ; \frac{\partial C_{s 2}}{\partial r}=0 \quad r=\delta_{1} ; \quad \frac{\partial C_{s 2}}{\partial r}=0 \quad r=\delta_{2} \quad t>0 \\
& P_{2}=\varepsilon_{2}+\rho k_{p 2}
\end{aligned}
$$

\subsection{Balanço na Membrana circular 2: Produto}

$$
\begin{aligned}
& P_{2} \frac{\partial C_{p 2}}{\partial t}-\nabla\left(D_{p 2} C_{p 2}\right)=R_{s} \quad t>0 \quad \delta_{1}<r<\delta_{2} \\
& C_{p 2}(r, t=0)=0 \quad t=0 \quad \delta_{1}<r<\delta_{2} ; \frac{\partial C_{p 2}}{\partial r}=0 \quad r=\delta_{1} ; \quad \frac{\partial C_{p 2}}{\partial r}=\frac{I(t)}{F \times n \times D_{p 2}} \quad r=\delta_{2} \quad t>0
\end{aligned}
$$

onde $\mathrm{C}_{\mathrm{p} 1}(\mathrm{r}, \mathrm{t})$ é o perfil de concentração de produto ao longo do raio da membrana circular $2 \mathrm{e}$ é medido por mol.m ${ }^{-3} ; \delta_{2}$ é o raio externo da membrana circular porosa $2, \mathrm{r}=\delta_{2}$, e é medido em $\mathrm{m} ; \mathrm{D}_{\mathrm{s} 2}$ é o coeficiente de difusão do efluente na membrana circular porosa 2 e é medido em $\mathrm{m}^{2} \cdot \mathrm{s}^{-1} ; \mathrm{D}_{\mathrm{p} 2}$ é o coeficiente de difusão do produto na membrana circular porosa 2 e é medido em $\mathrm{m}^{2} \cdot \mathrm{s}^{-1} ; \mathrm{I}(\mathrm{t})$ é a densidade de corrente de saída do eletrodo de trabalho $\mathrm{A} / \mathrm{m}^{2}$; F é a constante de Faraday em C.mol ${ }^{-1}$, n é o número de elétrons; $\mathrm{R}_{\mathrm{S}}$ é a lei de velocidade de reação de reação conforme o modelo de reação enzimática de Michaeles-Menten, e é dada pela Eq. 1.7, e é medida como mol. $\mathrm{m}^{-3} \cdot \mathrm{s}^{-1}$ :

$$
R_{s}=\mu_{\max } \frac{C_{s 2}}{K_{m}+C_{s 2}}
$$

no qual $\mu_{\max }$ é a constante de velocidade máxima dada por $\mathrm{s}^{-1}, \mathrm{~K}_{\mathrm{m}}$ é constante de Michaelis dada por mol. $\mathrm{m}^{-3}$.

\subsection{Balanço de carga no eletrodo de nanopartícula de cobre:}

A densidade de corrente de saída do eletrodo de trabalho I ( $t$ ) depende do fluxo de elétrons da reação de oxi-redução que acontece na interface entre membrana circular porosa 2 e o eletrodo de trabalho em $\mathrm{r}=\delta_{2}$. Nessa interface, também há interferência da espécie mediadora, produto $\mathrm{P}_{1}$, o qual é eliminado na superfície do eletrodo. Utilizou-se a lei de Faraday e a lei de Fick para expressar a densidade de corrente de saída, I (t), do fluxo de elétrons no eletrodo quando este é oxidado no eletrodo de trabalho:

$$
I(t)=n \times F \times D_{p 1 m 2} \times\left.\frac{\partial P_{1}}{\partial x}\right|_{x=\delta_{2}}
$$




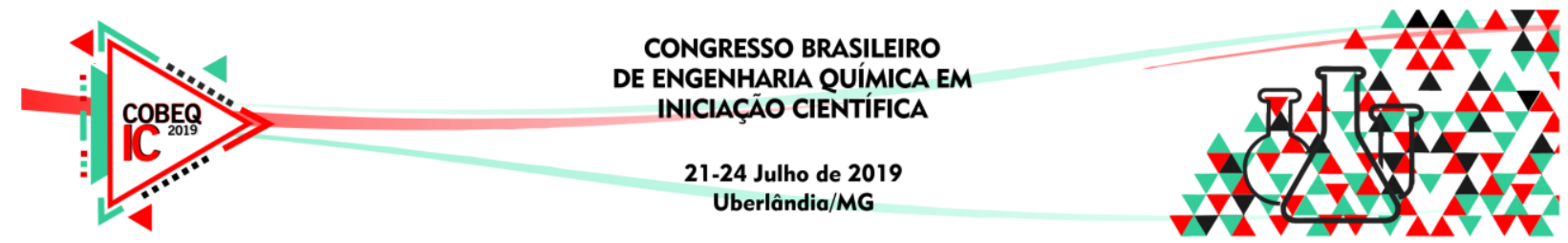

\section{RESULTADOS E DISCUSSÕES}

Os resultados foram obtidos através das simulações com o COMSOL MULTIPHISICS e foram utilizados os valores apresentados na Tabela 1 para as simulações.

Tabela 1 - Parâmetros utilizados na simulação.

\begin{tabular}{|c|c|c|c|c|c|}
\hline $\begin{array}{c}\mathbf{D}_{\text {s2 }} \\
\mathrm{m}^{2} / \mathrm{s}\end{array}$ & $\begin{array}{c}\boldsymbol{\delta}_{\mathbf{1}} \\
\mathrm{m}\end{array}$ & $\begin{array}{c}\boldsymbol{\delta}_{\mathbf{2}} \\
\mathrm{m}\end{array}$ & $\begin{array}{c}\boldsymbol{\mu}_{\max } \\
1 / \mathrm{s}\end{array}$ & $\begin{array}{c}\mathbf{K}_{\mathbf{m}} \\
\mathrm{mol} / \mathrm{m}^{3}\end{array}$ & $\begin{array}{c}\mathbf{n} \\
\text { s.u. }\end{array}$ \\
\hline $0,1 \times \mathrm{D}_{\mathrm{s} 1}$ & $5 \times 10^{-3}$ & $1 \times 10^{-2}$ & $4,83 \times 10^{-4}$ & $2,99 \times 10^{-4}$ & 2 \\
\hline
\end{tabular}

A simulação foi dividida em três estudos baseados em distintos valores de porosidade $\varepsilon_{2}$ na membrana porosa 2, e apresentados no Quadros 1 a 3. A porosidade na primeira e na segunda membrana variou em $\varepsilon_{1}=\varepsilon_{2}=0.1 ; 0.5$ e 0.9 . Todos os ensaios, representados pelas curvas presentes nos gráficos, foram feitos na faixa de tempo de 0 a 1 segundos. Os valores de $\mathrm{k}_{\mathrm{p} 1}$ e $\mathrm{k}_{\mathrm{p} 2}$ foram $10^{3}$ e $10^{-3}$, respectivamente; e os valores de $\mathrm{K}_{\mathrm{L}}$ foram considerados: $\mathrm{K}_{\mathrm{L}, \mathrm{s} 2}=$ $\mathrm{K}_{\mathrm{L}, \mathrm{s} 1}=0,001 \mathrm{~m}^{3} \cdot \mathrm{mol}^{-1}$ e $\mathrm{K}_{\mathrm{L}, \mathrm{pl}}=0,1 \mathrm{~m}^{3} \cdot \mathrm{mol}^{-1}$.

No primeiro estudo (Quadro 1), a porosidade adotada para a membrana 2, que possui a enzima imobilizada, foi de $\varepsilon_{2}=0.1 \times \varepsilon_{1}$, a partir das simulações para esse caso, observou-se que a porosidade influenciou de maneira satisfatória quanto à adsorção do efluente, onde a concentração diminui à medida que se expande ao longo do raio da membrana externa. A concentração de $10 \mathrm{~mol} / \mathrm{m}^{3}$ se mostrou com resultados mais satisfatórios.

No segundo estudo (Quadro 2), foram realizados os mesmos testes, porém utilizando a porosidade $\varepsilon_{2}=0,5 \times \varepsilon_{1}$. Nesse estudo, da mesma forma em relação ao anterior, a concentração de $S_{0}=10 \mathrm{~mol} / \mathrm{m}^{3}$ se mostrou mais satisfatória, enquanto que a concentração de $0,1 \mathrm{~mol} / \mathrm{m}^{3}$ teve um pico negativo próximo da posição de $0,055 \mathrm{~m}$ do raio externo, demonstrando que a concentração diminuiu a medida que se expandiu radialmente na membrana biopolimérica com enzima imobilizada.

No terceiro, e último (Quadro 3), estudo, a porosidade foi $\varepsilon_{2}=0,75 \times \varepsilon_{1}$, e realizou-se os mesmo estudos, observando a maneira que a reação se comporta no processo de reação do efluente com a membrana biopolimérica. Embora os valores utilizados tenham sido diferentes, os resultados se mantiveram num padrão, demonstrando que a porosidade influencia de maneira significativa na reação à medida que o efluente avança através das membranas 1 e 2 . Nesse caso, a concentração de efluente $S_{0}=10 \mathrm{~mol} / \mathrm{m}^{3}$ foi a melhor observada ao serem analisados os gráficos, sendo que a maior concentração inicial demonstrada pela reação entre substrato e membrana é representada pelo processo em que $\varepsilon_{1}$ é 0,1 , e a concentração é de $0,85 \mathrm{~mol} / \mathrm{m}^{3}$. 
Figura 2 - Simulações da influência da concentração de efluentes no processo difusivo poroso na membrana circular porosa $2\left(\varepsilon_{2}=0.1 \times \varepsilon_{1}\right)$.

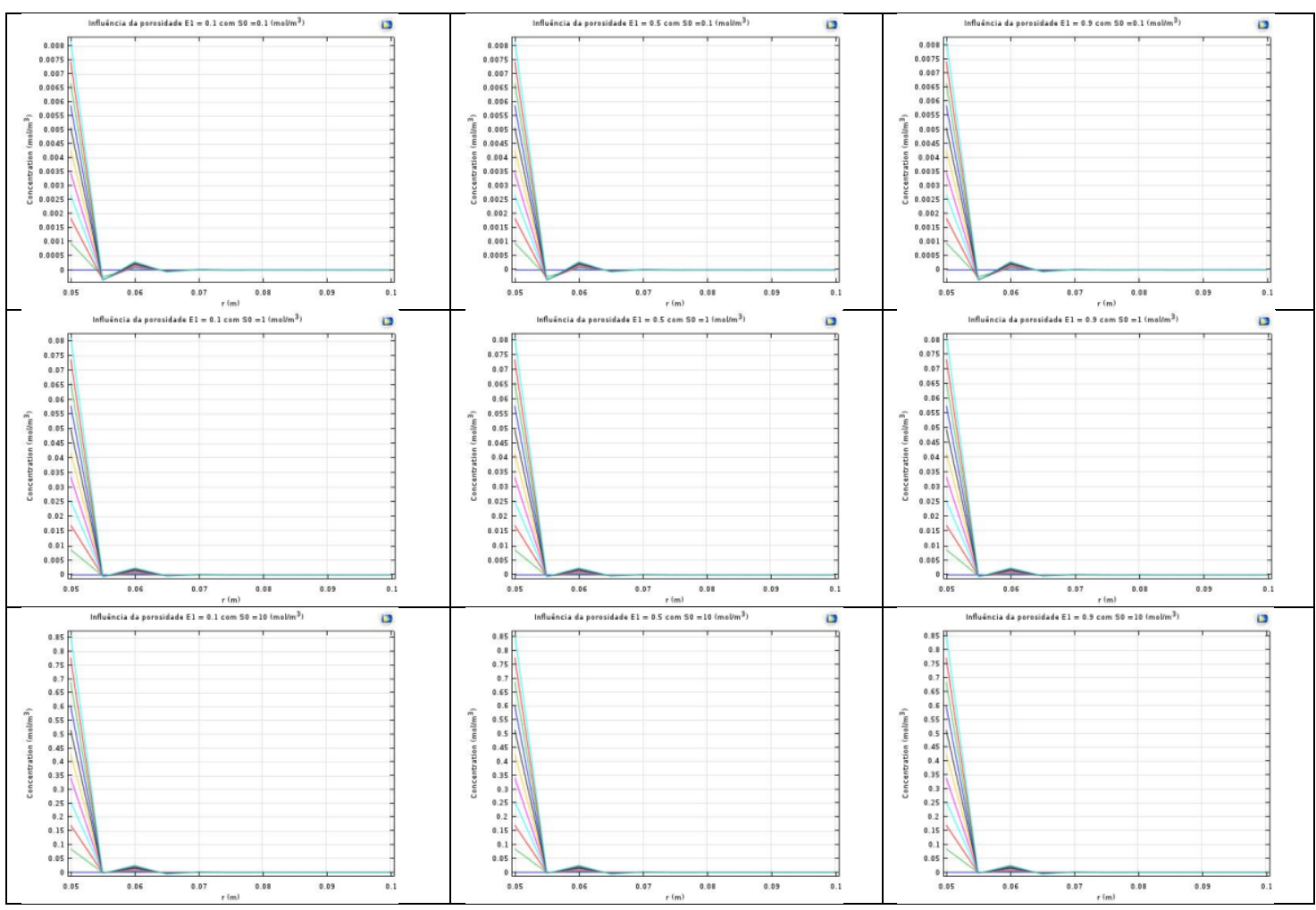

Figura 3 - Simulações da influência da concentração de efluentes no processo difusivo poroso na membrana circular porosa $2\left(\varepsilon_{2}=0.5 \times \varepsilon_{1}\right)$.

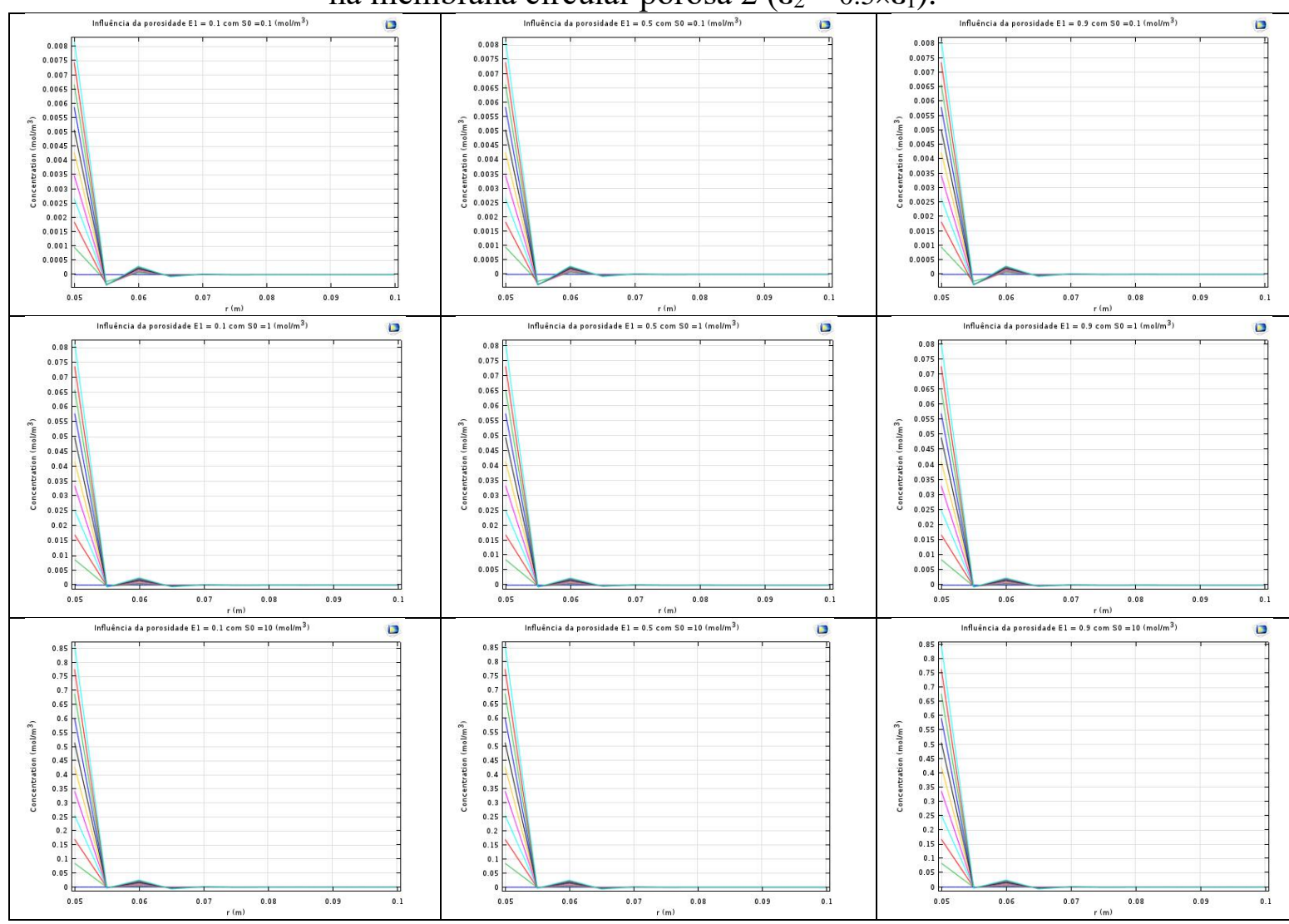




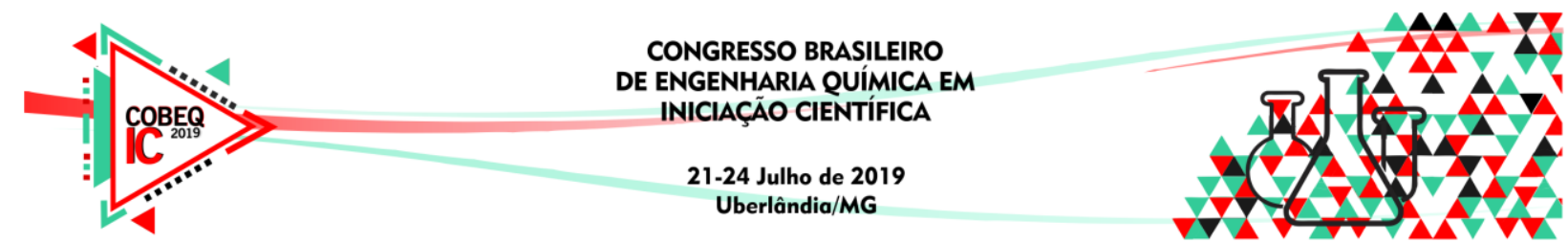

Figura 4 - Simulações da influência da concentração de efluentes no processo difusivo poroso na membrana circular porosa $2\left(\varepsilon_{2}=0.75 \varepsilon_{1}\right)$

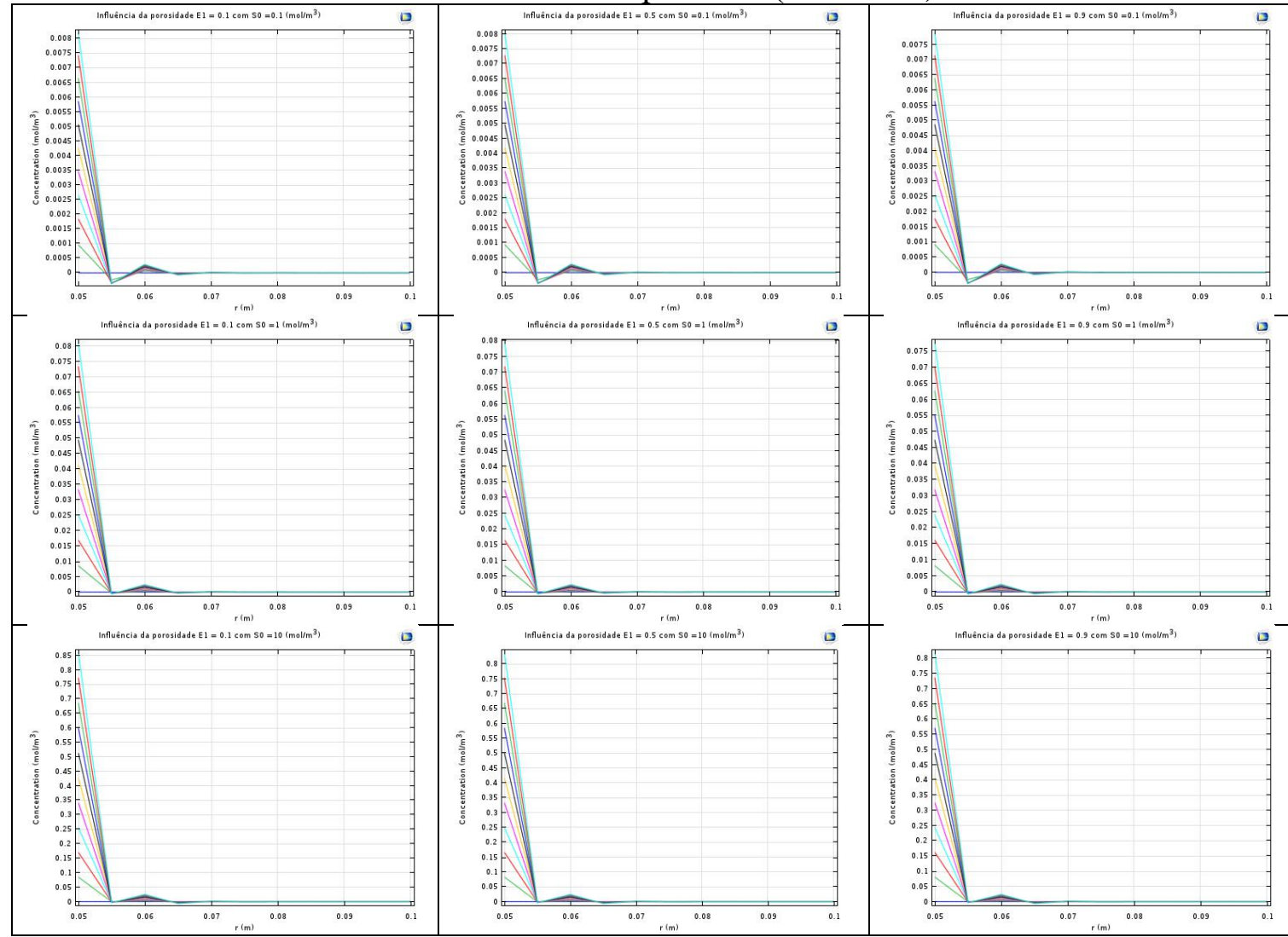

\section{CONCLUSÕES}

A partir do estudo realizado observou-se que a porosidade $\varepsilon_{2}$ influencia diretamente no processo de reação enzimática entre o substrato e a enzima para a detecção do fenol. Sendo assim, comprovou-se à interferência da porosidade nesse processo, onde a reação entre enzima e substrato na membrana porosa apresenta a concentração de efluente decrescendo a medida que se expande de maneira radial na superfície do biossensor, justificando que a porosidade auxilia no processo de adsorção do efluente (necessário para a identificação dos compostos fenólicos), junto à camada biopolimérica com enzima imobilizada e apresentando poros em sua extensão no biossensor.

\section{REFERÊNCIAS}

\section{CALIL, S. S.; SILVA, P. R. Q. Biossensores: estrutura, funcionamento e aplicabilidade.}

CARDOSO, E. T. C. Inibição da Atividade da Tirosinase por Análogos do Ácido Kójico. Universidade Federal do Pará, 2014.

NEGRI, T. C.; B., P. R. de A.; BRAZACA, S. G. C. Nutritional Value of Native and Exotic Fruits from Brazil. Biosaúde, Londrina, v. 18, n. 2, 2016.

OLIVEIRA, D. P. C., RIBEIRO, F. W. P., BECKER, H. et al. An Electrochemical Biosensor Based on The Tyrosinase Enzyme for The Determination of Phenol in Wastewater. Departamento de Química Analítica e Físico-Química, Centro de Ciências, Universidade Federal do Ceará, 2015. 